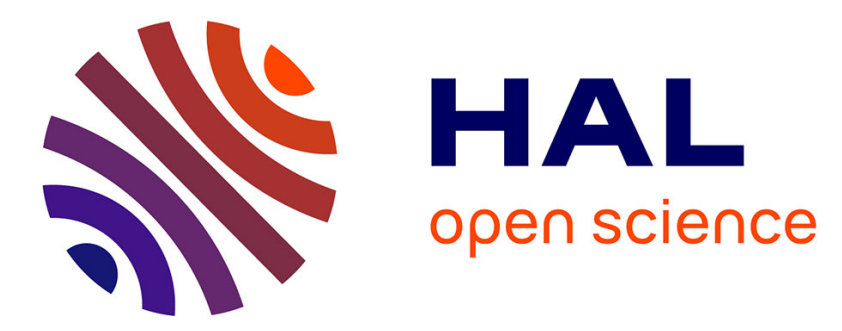

\title{
Quasi-hydrostatic primitive equations for ocean global circulation models
}

\author{
Carine Lucas, Madalina Petcu, Antoine Rousseau
}

\section{To cite this version:}

Carine Lucas, Madalina Petcu, Antoine Rousseau. Quasi-hydrostatic primitive equations for ocean global circulation models. Chinese Annals of Mathematics - Series B, 2010, 31 (6), pp.939-952. 10.1007/s11401-010-0611-6 . hal-00544799

\section{HAL Id: hal-00544799 \\ https://hal.science/hal-00544799}

Submitted on 9 Dec 2010

HAL is a multi-disciplinary open access archive for the deposit and dissemination of scientific research documents, whether they are published or not. The documents may come from teaching and research institutions in France or abroad, or from public or private research centers.
L'archive ouverte pluridisciplinaire HAL, est destinée au dépôt et à la diffusion de documents scientifiques de niveau recherche, publiés ou non, émanant des établissements d'enseignement et de recherche français ou étrangers, des laboratoires publics ou privés. 


\title{
Quasi-hydrostatic primitive equations for ocean global circulation models
}

\author{
Carine LUCAS ${ }^{1} \quad$ Madalina PETCU ${ }^{2} \quad$ Antoine ROUSSEAU ${ }^{3}$ \\ (Dedicated to Professor Roger Temam on the Occasion of his 70th Birthday)
}

\begin{abstract}
Global existence of weak and strong solutions to the quasi-hydrostatic primitive equations is studied in this paper. This model, that derives from the full non-hydrostatic model for geophysical fluid dynamics in the zero-limit of the aspect ratio, is more realistic than the classical hydrostatic model, since the traditional approximation that consists in neglecting a part of the Coriolis force is relaxed. After justifying the derivation of the model, we provide a rigorous proof of global existence of weak solutions, and well-posedness for strong solutions in dimension three.
\end{abstract}

Keywords Hydrostatic approximation, Coriolis force, ocean global circulation models, primitive equations, traditional approximation.

2000 MR Subject Classification 76M45, 76U05, 35B40, 35Q35, 76M20.

\section{Introduction}

Numerical models for the simulation of ocean global circulation seek to simulate fluid flows with the best compromise between computational cost and physical representativity. On the one hand, the complete Navier-Stokes equations (NSE), that contain all the required dynamical processes for a sharp modeling of the physics, is unfortunately too costly to be implemented at a high resolution on a wide computational domain. On the other hand, the hydrostatic primitive equations (HPEs) provide a competitively economic model (and yet physically satisfying) that is widely used for ocean global circulation (see for example ROMS and OPA models, [SM05] and [MDIL99, Mad08]).

The HPEs (see [Ped87, CR94, Gil82]) are simpler in several respects than the complete equations of motion. They rely on the smallness of the aspect ratio $\varepsilon=H / L$ (where $H$ and $L$ represent the typical height and length of the computational domain), $\varepsilon$ being typically of order $10^{-3}$ for large scale ocean models (see Table 1 below). In addition to the neglect of vertical accelerations in the momentum equations, the HPEs also include the so-called traditional approximation, which omits the Coriolis terms involving $2 \Omega \cos \theta$ that appear in the zonal and vertical components of the momentum equation.

\footnotetext{
Manuscript received May 31, 2010

${ }^{1}$ Laboratoire MAPMO (UMR 6628), Fédération Denis Poisson (FDP-FR2964), Université d'Orléans, Bâtiment de mathématiques - Route de Chartres, B.P. 6759, 45067 Orléans Cedex 2, France.

E-mail: Carine.Lucasuniv-orleans.fr

${ }^{2}$ Laboratoire de Mathématiques et Applications, Téléport 2 - BP 30179, Boulevard Marie et Pierre Curie, 86962 Futuroscope Chasseneuil Cedex, France. E-mail: Madalina.Petcu@math.univ-poitiers.fr

${ }^{3}$ INRIA,Laboratoire Jean Kuntzmann, 51 rue des mathématiques, B.P. 53, 38041 Grenoble Cedex 9, France. E-mail: Antoine.Rousseau@inria.fr
} 
This traditional approximation has been a matter of controversy for many years (see [Eck60], and the discussion initiated by Phillips [Phi66, Ver68, Phi68, Wan70]), and more recently it has been rigorously considered in physical studies (see [WB95, WHRS05]). Some of the authors have also evidenced in [LR08, LR10] the role played by the traditional approximation in simpler models such as Saint-Venant or quasi-geostrophic equations, for which numerical simulations confirm the existing differences between models based (or not) on the traditional approximation. The conclusion is that the omitted terms may be non-negligible in planetary-scale motion, and it is thus of interest to consider the HPEs from which the traditional approximation is removed. These new equations, that take the $2 \Omega \cos \theta$ terms into account, are called the quasi-hydrostatic primitive equations (QHPEs) and we want to dedicate this paper to their mathematical study.

From the mathematical viewpoint, there exists a large gap between the HPEs and the complete NSE. In the 90's, after pioneering works on the HPEs (see [LTW92a, LTW92b]), people were convinced that the hydrostatic model, because of the loss of the $\partial w / \partial t$ term, was even more challenging that the NSE. Roughly speaking, the vertical velocity $w$ in the HPEs becomes a diagnostic variable, computed a posteriori thanks to the divergence free equation (of course, we consider incompressible fluids). It was thus widely admitted that the corresponding loss of regularity for $w$ was a critical issue.

Surprisingly, the HPEs are better suited than expected. In Cao and Titi [CT07] and Kobelkov [Kob07], the authors proved a striking result with a global existence theorem for the strong solutions of the HPEs. The key point is to take advantage of the quasi-2D structure of the pressure to get rid of it and bring back the equations to a sort of viscous Burgers equation. Since 2007, the results of Cao, Titi and Kobelkov have been slightly improved (with less demanding hypotheses on the domain, see [KZ07]) and the review paper [PTZ08] is dedicated to the recent history of the HPEs.

In the following, we want to extend the above results to the QHPEs, for which no theoretical study has ever been published to the best of our knowledge (see however [DS05, LR08, LR10] for the role of $2 \Omega \cos \theta$ Coriolis terms in more simple models).

The paper is organized as follows: in Section 2, we present the derivation of the QHPEs, thanks to an asymptotic analysis that underlines the potential inconsistency of the traditional approximation. Then, we present in Section 3 a rigorous mathematical analysis of the corresponding initial-boundary value problem, extending the results of previous authors on the HPEs. A distinction between weak and strong solutions is done, as for the review paper [PTZ08]. 


\section{Derivation of the Model}

We start with the full equations of ocean dynamics:

$$
\begin{aligned}
\frac{\partial u}{\partial t}+\left(\mathbf{V}_{3} \cdot \nabla\right) u-f v+f^{*} w+\frac{\partial \phi}{\partial x}-\mu_{\mathbf{v}} \Delta_{h} u-\nu_{\mathbf{v}} \frac{\partial^{2} u}{\partial z^{2}} & =0 \\
\frac{\partial v}{\partial t}+\left(\mathbf{V}_{3} \cdot \nabla\right) v+f u+\frac{\partial \phi}{\partial y}-\mu_{\mathbf{v}} \Delta_{h} v-\nu_{\mathbf{v}} \frac{\partial^{2} v}{\partial z^{2}} & =0 \\
\frac{\partial w}{\partial t}+\left(\mathbf{V}_{3} \cdot \nabla\right) w-f^{*} u+\frac{\partial \phi}{\partial z}-\mu_{\mathbf{v}} \Delta_{h} w-\nu_{\mathbf{v}} \frac{\partial^{2} w}{\partial z^{2}} & =-\frac{\rho}{\rho_{0}} g, \\
\frac{\partial u}{\partial x}+\frac{\partial v}{\partial y}+\frac{\partial w}{\partial z} & =0 \\
\frac{\partial T}{\partial t}+\left(\mathbf{V}_{3} \cdot \nabla\right) T-\mu_{T} \Delta_{h} T-\nu_{T} \frac{\partial^{2} T}{\partial z^{2}} & =F_{T}, \\
\frac{\partial S}{\partial t}+\left(\mathbf{V}_{3} \cdot \nabla\right) S-\mu_{S} \Delta_{h} S-\nu_{S} \frac{\partial^{2} S}{\partial z^{2}} & =0 .
\end{aligned}
$$

Here $\mathbf{V}_{3}=(u, v, w)=(\mathbf{v}, w), \rho, T$ and $S$ are respectively the three-dimensional velocity, density, temperature and salinity of the fluid, and $\phi$ is the renormalized pressure, $\phi=p / \rho_{0}$. The constant numbers $f=2 \Omega \sin (\theta)$ and $f^{*}=2 \Omega \cos (\theta)$ are the Coriolis parameters. The vector $\vec{\Omega}=\Omega(\cos (\theta), \sin (\theta), 0)$ represents the Earth's rotation at the constant latitude $\theta, g$ is the universal gravity constant, and $\rho_{0}$ stands for the averaged density of the fluid. Finally, $\left(\mu_{\mathbf{v}}, \mu_{T}, \mu_{S}\right)$ and $\left(\nu_{\mathbf{v}}, \nu_{T}, \nu_{S}\right)$ are the horizontal and vertical viscosities, and $F_{T}$ represent the external heating term. For the sake of simplicity and since it does not raise any additional mathematical difficulty, $F_{T}$ will be set to zero in the sequel.

This model is closed by the state equation that describes the relationship between density, temperature and salinity. We will consider a linear dependency of $\rho$ with respect to $T$ and $S$ (the linear dependency between $\rho, T$ and $S$ is widely admitted in the literature (see articles cited above)):

$$
\rho(T, S)=\rho_{0}\left(1-\beta_{T}\left(T-T^{*}\right)+\beta_{S}\left(S-S^{*}\right)\right)
$$

where $\beta_{T}$ and $\beta_{S}$ are two positive constants.

The model (2.1) relies on a fundamental approximation on the density: the Boussinesq approximation. It consists in considering the water density as constant $\left(\rho_{0}\right)$ in the momentum equations, except in the gravity term of (2.1c) where its variations are taken into account. Apart from the Boussinesq approximation on the density of the fluid, numerous other approximations have been proposed, discussed and implemented in the literature. Among others, the hydrostatic approximation consists in replacing $(2.1 \mathrm{c})$ by

$$
\frac{\partial \phi}{\partial z}=-\frac{\rho}{\rho_{0}} g,
$$

and is one of the most important, leading to the so-called hydrostatic primitive equations (HPEs) of the ocean. In this section, we will present and justify a different set of equations, called the quasi-hydrostatic primitive equations (QHPEs), from which the hydrostatic approximation has been removed, and replaced by a weaker (less demanding) approximation. 
Thanks to a scale analysis based on Table 1 below, we will explain why the $\cos \theta$ Coriolis terms cannot comfortably be neglected in global circulation models. The interested reader is referred to [WB95, WHRS05] and references therein for additional details on the physical phenomena involved in the various approximations.

\subsection{Orders of Magnitude}

In order to perform a scale analysis and discriminate between the terms that can be neglected and those that should be retained, we list in Table 1 the values that we consider for the physical parameters (length and height of the domain, earth's rotation angular velocity, vertical and horizontal velocities, etc.). These orders of magnitude typically correspond to a planetary-scale motion ( $W L / U H \approx 1$, see [Bur91]), for example to the realistic configuration of the Northern Atlantic Ocean.

\begin{tabular}{|c|c|}
\hline Height $(H)$ & $1000 \mathrm{~m}$ \\
\hline Length $(L)$ & $1000 \mathrm{~km}$ \\
\hline Horizontal Velocity $(U)$ & $1.0{\mathrm{~m} . \mathrm{s}^{-1}}^{-1}$ \\
\hline Vertical Velocity $(W)$ & $10^{-3} \mathrm{m.s}^{-1}$ \\
\hline Time $(T=L / U)$ & $10^{6} \mathrm{~s}$ \\
\hline Earth rot. velocity $(\Omega)$ & $7.10^{-5}{\mathrm{rad} . \mathrm{s}^{-1}}$
\end{tabular}

Table 1: Typical orders of magnitude for the Northern Atlantic Ocean.

Given those values, the aspect ratio $\varepsilon=H / L(=W / U)=10^{-3}$ denotes the strong shallowness of the considered domain.

\subsection{Traditional Hydrostatic Approximation}

Let us compare the material derivative $D w / D t=\partial w / \partial t+(U . \nabla) w$ to the Coriolis term $f^{*} u$ in the vertical momentum equation (2.1c). Thanks to Table 1 above, the ratio between these two terms ranges like

$$
\frac{W / T}{2 \Omega \cos \theta U}=\frac{\varepsilon}{T 2 \Omega \cos \theta}<\varepsilon
$$

It is hence justified to neglect the vertical acceleration in (2.1c).

A naive comparison between $f^{*} w$ and $f v$ in the zonal equation (2.1a) rapidly leads to the so-called traditional approximation and to the hydrostatic primitive equations: indeed, since $W$ scales as $\varepsilon U$, the $\cos \theta$ Coriolis term is neglected in the zonal equation, leading to the withdrawal of the term $f^{*} u$ in the vertical equation for conservation purposes. We finally come 
to the following (hydrostatic) primitive equations:

$$
\begin{aligned}
\frac{\partial u}{\partial t}+\left(\mathbf{V}_{3} \cdot \nabla\right) u-f v+\frac{\partial \phi}{\partial x}-\mu_{\mathbf{v}} \Delta_{h} u-\nu_{\mathbf{v}} \frac{\partial^{2} u}{\partial z^{2}} & =0 \\
\frac{\partial v}{\partial t}+\left(\mathbf{V}_{3} \cdot \nabla\right) v+f u+\frac{\partial \phi}{\partial y}-\mu_{\mathbf{v}} \Delta_{h} v-\nu_{\mathbf{v}} \frac{\partial^{2} v}{\partial z^{2}} & =0 \\
\frac{\partial \phi}{\partial z} & =-\frac{\rho}{\rho_{0}} g, \\
\frac{\partial u}{\partial x}+\frac{\partial v}{\partial y}+\frac{\partial w}{\partial z} & =0 \\
\frac{\partial T}{\partial t}+\left(\mathbf{V}_{3} \cdot \nabla\right) T-\mu_{T} \Delta_{h} T-\nu_{T} \frac{\partial^{2} T}{\partial z^{2}} & =0 . \\
\frac{\partial S}{\partial t}+\left(\mathbf{V}_{3} \cdot \nabla\right) S-\mu_{S} \Delta_{h} S-\nu_{S} \frac{\partial^{2} S}{\partial z^{2}} & =0 .
\end{aligned}
$$

Over the last decades, this model has been widely used by oceanographers for operational computations and studied by applied mathematicians (see references in Section 1 above).

\subsection{Importance of the $\cos \theta$ Coriolis terms}

We want to show in the sequel that the $\cos \theta$ Coriolis terms are by far the largest of the omitted terms. The following alternate scale analysis, together with the theoretical clues for the well-posedness of the corresponding mathematical problem, makes us believe that the quasihydrostatic primitive equations realize the best compromize between physical representativeness and mathematical solvability.

Let us proceed to an alternate scale analysis of the zonal equation, and consider the term $f^{*} w$ in relation to the material derivative $D u / D t$ in the zonal equation (2.1a). The ratio scales like

$$
\frac{2 \Omega \cos \theta W}{U / T}=\frac{2 \varepsilon \Omega}{T} \cos \theta=14 \% \cos \theta
$$

and thus the retention of the term $f^{*} w$ seems desirable. For conservation purposes, it is also desirable to retain the $2 \Omega \cos \theta u$ term in the vertical momentum equation (2.1c) (it can also be shown that the $2 \Omega \cos \theta u$ term may be retained in Equation (2.1c) (see [WB95]) regardless of conservation arguments, see Remark 2.1), so that the Coriolis force remains orthogonal to the fluid velocity. We finally end up with the following quasi-hydrostatic primitive equations:

$$
\begin{aligned}
\frac{\partial u}{\partial t}+\left(\mathbf{V}_{3} \cdot \nabla\right) u-f v+f^{*} w+\frac{\partial \phi}{\partial x}-\mu_{\mathbf{v}} \Delta_{h} u-\nu_{\mathbf{v}} \frac{\partial^{2} u}{\partial z^{2}} & =0 \\
\frac{\partial v}{\partial t}+\left(\mathbf{V}_{3} \cdot \nabla\right) v+f u+\frac{\partial \phi}{\partial y}-\mu_{\mathbf{v}} \Delta_{h} v-\nu_{\mathbf{v}} \frac{\partial^{2} v}{\partial z^{2}} & =0 \\
-f^{*} u+\frac{\partial \phi}{\partial z} & =-\frac{\rho}{\rho_{0}} g, \\
\frac{\partial u}{\partial x}+\frac{\partial v}{\partial y}+\frac{\partial w}{\partial z} & =0 \\
\frac{\partial T}{\partial t}+\left(\mathbf{V}_{3} \cdot \nabla\right) T-\mu_{T} \Delta_{h} T-\nu_{T} \frac{\partial^{2} T}{\partial z^{2}} & =0 . \\
\frac{\partial S}{\partial t}+\left(\mathbf{V}_{3} \cdot \nabla\right) S-\mu_{S} \Delta_{h} S-\nu_{S} \frac{\partial^{2} S}{\partial z^{2}} & =0 .
\end{aligned}
$$


In Section 3 below, we investigate the mathematical properties of the QHPEs. In particular, we extend the global existence results obtained in [CT07, Kob07, PTZ08] both for weak and strong solutions of the HPEs. As the salinity and temperature equations have the same structure (and can thus be similarly considered from the mathematical viewpoint), we forget Equation (2.7f) with no loss of generality.

Remark 2.1 Importance of the $2 \Omega \cos \theta u$ in Equation (2.1c) Regardless of conservation arguments, we can also justify the retention of the $f^{*} u$ term in Equation (2.1c). Indeed, the ratio between this term and the pressure vertical derivative scales like

$$
\frac{2 \Omega \cos \theta U}{P / \rho H}=\frac{2 \Omega \cos \theta U}{U \Omega / \varepsilon}=2 \cos \theta \varepsilon
$$

which is actually small, but not as small as the ratio between $D w / D t$ and the pressure vertical derivative, which scales like $\varepsilon^{2} / 70$. In a way, we can say that the QHPEs consist in the conservation of terms of order 0 and order $\varepsilon$, getting rid of the $\varepsilon^{2}$ terms...

\subsection{Quasi-Hydrostatic Model: the Initial Boundary Value Problem}

Let us consider the QHPEs (2.7) on a cylindrical domain: $\mathcal{M}=\mathcal{M}^{\prime} \times(-h, 0)$ (see Figure 1 below), where $\mathcal{M}^{\prime}$ is a smooth bounded domain of $\mathbb{R}^{2}$. We denote by $\Gamma_{i}$ the surface at $z=0, \Gamma_{b}$ the bottom at $z=-h$, and $\Gamma_{\ell}$ the lateral surface. We also define $\boldsymbol{n}=\left(\boldsymbol{n}_{h}, \boldsymbol{n}_{z}\right)$ the unit outward normal on the boundary of the domain. We supplement equations (2.7) with the following

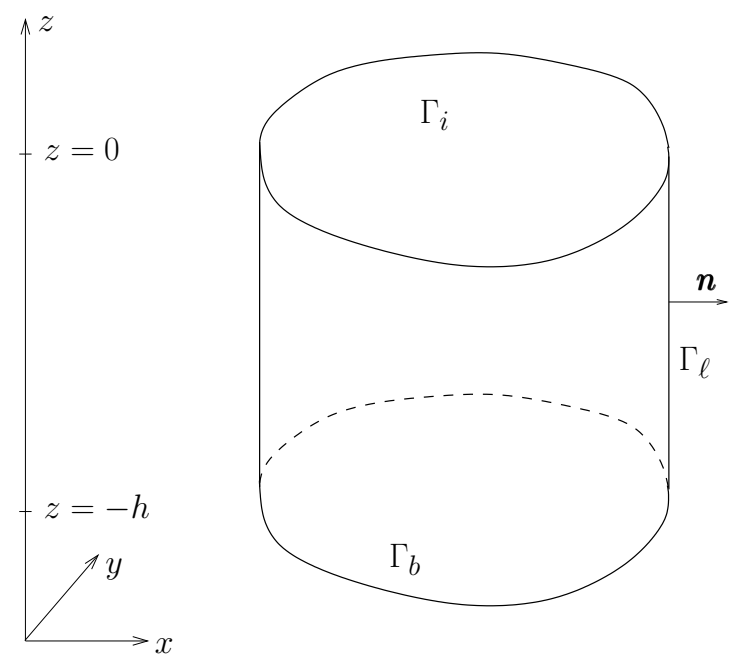

Figure 1: Cylindrical domain on which we solve the QHPEs.

boundary conditions (wind driven on the top surface and no-slip non-heat flux on side walls 
and bottom):

$$
\begin{aligned}
& \text { on } \Gamma_{i}: \frac{\partial \mathbf{v}}{\partial z}=h \tau, w=0, \frac{\partial T}{\partial z}=-\alpha_{T}\left(T-T^{*}\right), \\
& \text { on } \Gamma_{b}: \frac{\partial \mathbf{v}}{\partial z}=0, w=0, \frac{\partial T}{\partial z}=0, \\
& \text { on } \Gamma_{l}: \mathbf{v} \cdot \boldsymbol{n}=0, \frac{\partial \mathbf{v}}{\partial \boldsymbol{n}} \times \boldsymbol{n}=0, \frac{\partial T}{\partial \boldsymbol{n}}=0,
\end{aligned}
$$

where $\tau(x, y)$ and $T^{*}(x, y)$ are the wind stress and a typical temperature distribution on the ocean surface. With no loss of generality (see [CT07, Remark 1]), we assume that $\tau=0$ and $T^{*}=0$, but our results remain valid for sufficiently smooth non-zero data. Taking $T^{*}=0$ corresponds to the study of the perturbed temperature (and hence we substract the hydrostatic equilibrium pressure from the definition of $\phi$ ). That is, taking into account the state equation (2.2), Equation $(2.7 \mathrm{c})$ becomes

$$
\frac{\partial \phi}{\partial z}=\beta_{T} T g+f^{*} u
$$

We also supply our equations with initial data:

$$
\begin{aligned}
& \mathbf{v}(t=0)=\mathbf{v}_{0}(x, y, z), \\
& T(t=0)=T_{0}(x, y, z),
\end{aligned}
$$

where $\mathbf{v}_{0}$ and $T_{0}$ belong to spaces to be defined later on.

\section{Well-Posedness of the QHPEs}

In this section, we first prove global existence of weak solutions of (2.7), before studying the question of strong solutions. These results are based on previous works on HPEs, and do not take the $\cos \theta$ Coriolis terms into account. After recalling the main step of the proofs, we focus on the estimates for the new terms.

\subsection{Weak Solutions}

In order to write the weak formulation of equations (2.7), we introduce some notations:

$$
\begin{aligned}
\mathbf{U}= & (u, v, T)=(\mathbf{v}, T) \\
V= & \left\{\mathbf{U} \in\left(H^{1}(\mathcal{M})\right)^{3} \text { s. t. } \int_{-h}^{0} \nabla_{h} \cdot \mathbf{v} d z=0, \mathbf{v}=0 \text { on } \Gamma_{b} \cup \Gamma_{\ell}\right\} \\
H= & \left\{\mathbf{U} \in\left(L^{2}(\mathcal{M})\right)^{3} \text { s. t. } \int_{-h}^{0} \nabla_{h} \cdot \mathbf{v} d z=0, \boldsymbol{n}_{h} \cdot \int_{-h}^{0} \mathbf{v} d z=0 \text { on } \Gamma_{\ell}\right\}, \\
\|\mathbf{U}\|= & ((\mathbf{U}, \mathbf{U}))^{1 / 2} \text { and }|\mathbf{U}|_{H}=(\mathbf{U}, \mathbf{U})_{H}^{1 / 2} \text { where } \\
((\mathbf{U}, \widetilde{\mathbf{U}}))= & \int_{\mathcal{M}}\left(\mu_{\mathbf{v}} \nabla_{h} \mathbf{v} \cdot \nabla_{h} \widetilde{\mathbf{v}}+\nu_{\mathbf{v}} \frac{\partial \mathbf{v}}{\partial z} \frac{\partial \widetilde{\mathbf{v}}}{\partial z}\right) d \mathcal{M} \\
& +K_{T} \int_{\mathcal{M}}\left(\mu_{T} \nabla_{h} T \cdot \nabla_{h} \widetilde{T}+\nu_{T} \frac{\partial T}{\partial z} \frac{\partial \widetilde{T}}{\partial z}\right) d \mathcal{M}+K_{T} \int_{\Gamma_{i}} \alpha_{T} T \widetilde{T} d \Gamma_{i}, \\
(\mathbf{U}, \widetilde{\mathbf{U}})_{H}= & \int_{\mathcal{M}}\left(\mathbf{v} \cdot \widetilde{\mathbf{v}}+K_{T} T \widetilde{T}\right) d \mathcal{M}
\end{aligned}
$$


where $K_{T}$ is an arbitrary positive constant.

We also denote by $\phi_{s}$ the renormalized pressure at the surface $z=0$, such that:

$$
\phi(x, y, z, t)=\phi_{s}(x, y, t)-\beta_{T} g \int_{z}^{0} T(x, y, \xi, t) d \xi-f^{*} \int_{z}^{0} u(x, y, \xi, t) d \xi,
$$

where we integrated Equation (2.10) in the vertical direction.

In the same way, integrating Equation (2.7d), we can express $w$ as a function of $\mathbf{v}$. The functions $\phi$ and $w$ are said to be diagnostic variables, contrarily to $\mathbf{U}$ which gathers the prognostic variables.

We consider a sufficiently regular test function $\widetilde{\mathbf{U}}=(\widetilde{\mathbf{v}}, \widetilde{T})=(\widetilde{u}, \widetilde{v}, \widetilde{T})$ in $V$ and we multiply Equation $(2.7 \mathrm{a})$ by $\widetilde{u}$, Equation $(2.7 \mathrm{~b})$ by $\widetilde{v}$, Equation $(2.7 \mathrm{e})$ by $\widetilde{T}$. We integrate over the domain $\mathcal{M}$ and find the weak formulation of the QHPEs:

$$
\begin{aligned}
& \left(\frac{d}{d t} \mathbf{U}, \widetilde{\mathbf{U}}\right)_{H}+a(\mathbf{U}, \widetilde{\mathbf{U}})+b(\mathbf{U}, \mathbf{U}, \widetilde{\mathbf{U}})+e(\mathbf{U}, \widetilde{\mathbf{U}})=0, \\
& \mathbf{U}(t=0)=\mathbf{U}_{0}
\end{aligned}
$$

with

$$
\begin{aligned}
a(\mathbf{U}, \widetilde{\mathbf{U}})= & ((\mathbf{U}, \widetilde{\mathbf{U}}))-\int_{\mathcal{M}} \phi_{s} \nabla_{h} \cdot \widetilde{\mathbf{v}} d \mathcal{M}+\beta_{T} g \int_{\mathcal{M}}\left(\int_{z}^{0} T(x, y, \xi, t) d \xi\right) \nabla_{h} \cdot \widetilde{\mathbf{v}} d \mathcal{M}, \\
b\left(\mathbf{U}, \widetilde{\mathbf{U}}, \mathbf{U}^{\sharp}\right)= & \int_{\mathcal{M}}\left(\mathbf{v} \cdot \nabla_{h} \widetilde{\mathbf{v}}+w(\mathbf{v}) \frac{\partial \widetilde{\mathbf{v}}}{\partial z}\right) \mathbf{v}^{\sharp} d \mathcal{M} \\
& +K_{T} \int_{\mathcal{M}}\left(\mathbf{v} \cdot \nabla_{h} \widetilde{T}+w(\mathbf{v}) \frac{\partial \widetilde{T}}{\partial z}\right) T^{\sharp} d \mathcal{M}, \\
e(\mathbf{U}, \widetilde{\mathbf{U}})= & f \int_{\mathcal{M}}(u \widetilde{v}-v \widetilde{u}) d \mathcal{M} \\
& +f^{*} \int_{\mathcal{M}}\left(\int_{z}^{0} u(x, y, \xi, t) d \xi\right) \nabla_{h} \cdot \widetilde{\mathbf{v}} d \mathcal{M}+f^{*} \int_{\mathcal{M}} w(\mathbf{v}) \widetilde{u} d \mathcal{M} .
\end{aligned}
$$

In [PTZ08, Section 2.2], the authors studied this system, without the $f^{*}$ terms in the expression of $e$. Their proof relies on finite differences in time, a priori estimates and passage to the limit. In particular, they need $a+e$ to be a coercive bilinear form and $b$ to be trilinear and continuous.

Here, as the bilinear form $a$ is not modified, its coercivity properties are still satisfied; for the trilinear form $b$ also we do not need any supplementary verification. The only point we detail in the following is the influence of the two new Coriolis terms in the expression of the bilinear form $e$.

Let us remark that, if

$$
I_{c}:=f^{*} \int_{\mathcal{M}}\left(\int_{z}^{0} u(x, y, \xi, t) d \xi\right) \nabla_{h} \cdot \mathbf{v} d \mathcal{M}+f^{*} \int_{\mathcal{M}} w(\mathbf{v}) u d \mathcal{M}
$$

we have (remember that the domain is a cylinder):

$$
\begin{aligned}
I_{c}= & f^{*} \int_{\mathcal{M}}\left(\int_{z}^{0} u(x, y, \xi, t) d \xi\right) \nabla_{h} \cdot \mathbf{v} d \mathcal{M}+f^{*} \int_{\mathcal{M}}\left(\int_{z}^{0} \nabla_{h} \cdot \mathbf{v}(x, y, \xi, t) d \xi\right) u d \mathcal{M}, \\
= & f^{*} \int_{\mathcal{M}^{\prime}} \int_{-h}^{0}\left(\int_{z}^{0} u(x, y, \xi, t) d \xi\right) \nabla_{h} \cdot \mathbf{v} d z d \mathcal{M}^{\prime} \\
& +f^{*} \int_{\mathcal{M}^{\prime}} \int_{-h}^{0}\left(\int_{z}^{0} \nabla_{h} \cdot \mathbf{v}(x, y, \xi, t) d \xi\right) u d z d \mathcal{M}^{\prime} .
\end{aligned}
$$


Thanks to an integration by parts on the vertical variable and using the boundary conditions on $\Gamma_{i}$ and $\Gamma_{b}$, we find $I_{c}=0$ so that $e(\mathbf{U}, \mathbf{U})$ is not modified compared to the expression given by M. Petcu, R. Temam and M. Ziane. Consequently, the bilinear form $e$ still satisfies the relation:

$$
e(\mathbf{U}, \mathbf{U})=0
$$

and $a+e$ is coercive. At this point, we are exactly in the same conditions as in [PTZ08], and the existence of weak solutions of the QHPEs follows immediately:

Theorem 3.1 Let $\mathcal{M}$ be a cylindrical domain, $t_{1}>0$ and $\mathbf{U}_{0} \in H$ the initial condition. Then there exists $\mathbf{U}=(u, v, T)$ solution of system (3.1) such that

$$
\mathbf{U}=(u, v, T) \in L^{\infty}\left(0, t_{1} ; H\right) \cap L^{2}\left(0, t_{1} ; V\right) .
$$

\section{$3.2 \quad$ Strong Solutions}

The existence of strong solutions of (2.7) can be proved in the same way as in [CT07] for the hydrostatic primitive equations (without the $\cos \phi$ terms of the Coriolis force). For the sake of clarity, let us first recall the main steps of this paper.

The idea of C. Cao and E. S. Titi is to give a bound on the $H^{1}$ norm of the velocity and the temperature, in order to obtain global existence in time. But the overestimation of the $H^{1}$ norms is not a straightforward consequence of an energy inequality. Indeed, they have to compute several a priori estimates, especially a $L^{6}$ inequality, to be able to write the sought relation.

In the case of the quasi-hydrostatic primitive equations (with complete Coriolis force), we can only consider the influence of the new terms on these inequalities, and try to obtain the same type of a priori bounds.

\subsubsection{Reformulation of the problem}

Let us reformulate system (2.7): integrating equation (2.7d) on the vertical and taking into consideration the boundary conditions, we have the following expression of vertical velocity

$$
w(x, y, z, t)=-\int_{-h}^{z} \nabla_{h} \cdot \mathbf{v}(x, y, \xi, t) d \xi
$$


100000010

Then we integrate equation (2.10) and replace the expression of $\phi$ in Equation (2.7a)-(2.7b) to obtain:

$$
\begin{gathered}
\frac{\partial \mathbf{v}}{\partial t}+\left(\mathbf{v} \cdot \nabla_{h}\right) \mathbf{v}-\left(\int_{-h}^{z} \nabla_{h} \cdot \mathbf{v}\right) \frac{\partial \mathbf{v}}{\partial z}+f \mathbf{k} \times \mathbf{v}+f^{*}\left(\begin{array}{c}
w \\
0
\end{array}\right) \\
+\nabla_{h}\left(\phi_{s}-\beta_{T} g \int_{z}^{0} T-f^{*} \int_{z}^{0} u\right)-\mu_{\mathbf{v}} \Delta_{h} \mathbf{v}-\nu_{\mathbf{v}} \frac{\partial^{2} \mathbf{v}}{\partial z^{2}}=0 \\
\frac{\partial T}{\partial t}+\left(\mathbf{v} \cdot \nabla_{h}\right) T-\left(\int_{-h}^{z} \nabla_{h} \cdot \mathbf{v}\right) \frac{\partial T}{\partial z}-\mu_{T} \Delta_{h} T-\nu_{T} \frac{\partial^{2} T}{\partial z^{2}}=0 \\
\left.\frac{\partial \mathbf{v}}{\partial z}\right|_{z=0}=0,\left.\quad \frac{\partial \mathbf{v}}{\partial z}\right|_{z=-h}=0,\left.\quad \mathbf{v} \cdot \boldsymbol{n}\right|_{\Gamma_{\ell}}=0, \quad \frac{\partial \mathbf{v}}{\partial \boldsymbol{n}} \times\left.\boldsymbol{n}\right|_{\Gamma_{\ell}}=0 \\
\left(\begin{array}{c}
\left.\frac{\partial T}{\partial z}+\alpha_{T} T\right)\left.\right|_{z=0}=0,\left.\quad \frac{\partial T}{\partial z}\right|_{z=-h}=0,\left.\quad \frac{\partial T}{\partial \boldsymbol{n}}\right|_{\Gamma_{\ell}}=0 \\
\mathbf{v}(x, y, z, t=0)=\mathbf{v}_{0}(x, y, z), \quad T(x, y, z, t=0)=T_{0}(x, y, z)
\end{array}\right.
\end{gathered}
$$

where $\phi_{s}(x, y, t)=\phi(x, y, z=0, t)$.

We denote by $V_{1}$ the closure space of $\left\{\mathbf{v} \in \mathcal{C}^{\infty}\right.$ satisfying (3.3c) $\}$ and $V_{2}$ is the closure space of $\left\{T \in \mathcal{C}^{\infty}\right.$ satisfying $\left.(3.3 \mathrm{~d})\right\}$ in $H^{1}(\mathcal{M})$.

We decompose the horizontal velocity $\mathbf{v}$ as the sum of its barotropic part $\overline{\mathbf{v}}$ (equal to the mean value of $\mathbf{v}$ on the vertical) and its baroclinic part $\widetilde{\mathbf{v}}$. We also write $w=\bar{w}+\widetilde{w}$ with $\bar{w}=\frac{1}{h} \int_{-h}^{0} w(x, y, z, t) d z$. Then the following equations hold:

$$
\begin{aligned}
\frac{\partial \overline{\mathbf{v}}}{\partial t}+\left(\overline{\mathbf{v}} \cdot \nabla_{h}\right) \overline{\mathbf{v}}+\overline{\left(\widetilde{\mathbf{v}} \cdot \nabla_{h}\right) \widetilde{\mathbf{v}}+\left(\nabla_{h} \cdot \widetilde{\mathbf{v}}\right) \widetilde{\mathbf{v}}}+f \mathbf{k} \times \overline{\mathbf{v}}+f^{*}\left(\begin{array}{c}
\bar{w} \\
0
\end{array}\right)-\mu_{\mathbf{v}} \Delta_{h} \overline{\mathbf{v}} & \\
+\nabla_{h}\left(\phi_{s}-\frac{\beta_{T} g}{h} \int_{-h}^{0}\left(\int_{z}^{0} T\right) d z-\frac{f^{*}}{h} \int_{-h}^{0}\left(\int_{z}^{0} u\right) d z\right) & =0 \\
\nabla_{h} \cdot \overline{\mathbf{v}} & =0 \\
\left.\overline{\mathbf{v}} \cdot \boldsymbol{n}\right|_{\Gamma_{\ell}}=0, \quad \frac{\partial \overline{\mathbf{v}}}{\partial \boldsymbol{n}} \times\left.\boldsymbol{n}\right|_{\Gamma_{\ell}} & =0
\end{aligned}
$$

and

$$
\begin{gathered}
\frac{\partial \widetilde{\mathbf{v}}}{\partial t}+\left(\widetilde{\mathbf{v}} \cdot \nabla_{h}\right) \widetilde{\mathbf{v}}+\left(\widetilde{\mathbf{v}} \cdot \nabla_{h}\right) \overline{\mathbf{v}}+\left(\overline{\mathbf{v}} \cdot \nabla_{h}\right) \widetilde{\mathbf{v}}-\overline{\left(\widetilde{\mathbf{v}} \cdot \nabla_{h}\right) \widetilde{\mathbf{v}}+\left(\nabla_{h} \cdot \widetilde{\mathbf{v}}\right) \widetilde{\mathbf{v}}} \\
-\left(\int_{-h}^{z} \nabla_{h} \cdot \widetilde{\mathbf{v}}\right) \frac{\partial \widetilde{\mathbf{v}}}{\partial z}+f \mathbf{k} \times \widetilde{\mathbf{v}}+f^{*}\left(\begin{array}{c}
\widetilde{w} \\
0
\end{array}\right)-\mu_{\mathbf{v}} \Delta_{h} \widetilde{\mathbf{v}}-\nu_{\mathbf{v}} \frac{\partial^{2} \widetilde{\mathbf{v}}}{\partial z^{2}} \\
-\nabla_{h}\left(\beta_{T} g \int_{z}^{0} T-\frac{\beta_{T} g}{h} \int_{-h}^{0}\left(\int_{z}^{0} T\right) d z+f^{*} \int_{z}^{0} u-\frac{f^{*}}{h} \int_{-h}^{0}\left(\int_{z}^{0} u\right) d z\right)=0 \\
\left.\frac{\partial \widetilde{\mathbf{v}}}{\partial z}\right|_{z=0}=0,\left.\quad \frac{\partial \widetilde{\mathbf{v}}}{\partial z}\right|_{z=-h}=0,\left.\quad \widetilde{\mathbf{v}} \cdot \boldsymbol{n}\right|_{\Gamma_{\ell}}=0, \quad \frac{\partial \widetilde{\mathbf{v}}}{\partial \boldsymbol{n}} \times\left.\boldsymbol{n}\right|_{\Gamma_{\ell}}=0 .
\end{gathered}
$$

In Equation (3.4a), compared to the HPEs, the difference appears from the two terms

$$
f^{*}\left(\begin{array}{c}
\bar{w} \\
0
\end{array}\right) \text { and } \frac{f^{*}}{h} \nabla_{h} \int_{-h}^{0}\left(\int_{z}^{0} u\right) d z .
$$

For $\widetilde{\mathbf{v}}$, in Equation (3.5a), the new terms are:

$$
f^{*}\left(\begin{array}{c}
\widetilde{w} \\
0
\end{array}\right) \quad \text { and } \quad \nabla_{h}\left(-f^{*} \int_{z}^{0} u+\frac{f^{*}}{h} \int_{-h}^{0}\left(\int_{z}^{0} u\right) d z\right) .
$$


First, one must note that the $L^{2}$ estimates are not modified by the new terms. So $\mathbf{v}$ is in $L^{\infty}\left(0, t_{\max } ;\left(L^{2}(\mathcal{M})\right)^{2}\right), \nabla \mathbf{v}$ is in $L^{1}\left(0, t_{\max } ;\left(L^{2}(\mathcal{M})\right)^{6}\right)$ and similar properties stand for $T$.

We can improve this result and prove uniform bounds on $\mathbf{U}=(\mathbf{v}, T)$ (see [PTZ08]). More precisely, there exists $t_{0}$ that only depends on $\mathbf{U}_{0}=\mathbf{U}_{\mid t=0}$, such that, for all $t \geq t_{0}$ and $r>0$ :

$$
\int_{t}^{t+r}\|\mathbf{U}(s)\|^{2} d s \leq K
$$

where $K$ is a constant that does not depend on $\mathbf{U}_{0}$ and $\|\mathbf{U}\|=((\mathbf{U}, \mathbf{U}))^{1 / 2}$ is defined by:

$$
\begin{aligned}
((\mathbf{U}, \widetilde{\mathbf{U}}))=\int_{\mathcal{M}}\left(\mu_{\mathbf{v}} \nabla_{h} \mathbf{v}\right. & \left.\cdot \nabla_{h} \widetilde{\mathbf{v}}+\nu_{\mathbf{v}} \frac{\partial \mathbf{v}}{\partial z} \frac{\partial \widetilde{\mathbf{v}}}{\partial z}\right) d \mathcal{M} \\
& +K_{T} \int_{\mathcal{M}}\left(\mu_{T} \nabla_{h} T \cdot \nabla_{h} \widetilde{T}+\nu_{T} \frac{\partial T}{\partial z} \frac{\partial \widetilde{T}}{\partial z}\right) d \mathcal{M}+K_{T} \int_{\Gamma_{i}} \alpha_{T} T \widetilde{T} d \Gamma_{i} .
\end{aligned}
$$

Next, we have to study step by step all the bounds that are necessary to obtain the existence of strong solutions in [CT07].

\subsection{2 $\quad L^{6}$ bounds on the horizontal velocity}

Lemma 3.1 Let $\widetilde{\mathbf{v}}$ be a solution of system (3.5). Then, for all $t \geq t_{0}+r$ (defined above), we have a time-uniform bound on the $L^{6}$ norm of $\widetilde{\mathbf{v}}$.

Proof. We multiply equation $(3.5 \mathrm{a})$ by $|\widetilde{\mathbf{v}}|^{4} \widetilde{\mathbf{v}}$ and integrate over the domain $\mathcal{M}$. Following [CT07], we obtain

$$
\begin{aligned}
\frac{1}{6} \frac{d\|\widetilde{\mathbf{v}}\|_{6}^{6}}{d t}+\frac{\mu_{\mathbf{v}}}{6} \int_{\mathcal{M}} & \left|\nabla_{h} \widetilde{\mathbf{v}}\right|^{2}|\widetilde{\mathbf{v}}|^{4} d \mathcal{M}+\frac{\nu_{\mathbf{v}}}{6} \int_{\mathcal{M}}|\widetilde{\mathbf{v}} z|^{2}|\widetilde{\mathbf{v}}|^{4} d \mathcal{M}+A_{1}-A_{2} \\
& \leq C\|\overline{\mathbf{v}}\|_{2}^{2}\left\|\nabla_{h} \overline{\mathbf{v}}\right\|_{2}^{2}\|\widetilde{\mathbf{v}}\|_{6}^{6}+C\|\widetilde{\mathbf{v}}\|_{6}^{6}\left\|\nabla_{h} \widetilde{\mathbf{v}}\right\|_{2}^{2}+C\|\bar{T}\|_{2}^{2}\left\|\nabla_{h} \bar{T}\right\|_{2}^{2}+C\|\widetilde{\mathbf{v}}\|_{2}^{2}\|\widetilde{\mathbf{v}}\|_{6}^{6},
\end{aligned}
$$

where the new Coriolis terms are:

$$
\begin{aligned}
& A_{1}:=f^{*} \int_{\mathcal{M}} \widetilde{w}|\widetilde{\mathbf{v}}|^{4} \widetilde{u} d \mathcal{M} \\
& A_{2}:=\int_{\mathcal{M}} \nabla_{h}\left(f^{*} \int_{z}^{0} u(x, y, \xi, t) d \xi-\frac{f^{*}}{h} \int_{-h}^{0}\left(\int_{z}^{0} u(x, y, \xi, t) d \xi\right) d z\right) \cdot|\widetilde{\mathbf{v}}|^{4} \widetilde{\mathbf{v}} d \mathcal{M} .
\end{aligned}
$$

Note that, for the sake of simplicity, we will always denote by $C$ a positive constant, but this constant may be different for each term.

First, let us study the contribution of the term $A_{1}$.

Replacing $\widetilde{w}$ by the difference between $w$ and its mean value, using the expression of $w$ as a function of $\mathbf{v}(3.2)$, and thanks to an integration by parts, we get:

$$
\begin{aligned}
& A_{1}=-f^{*} \int_{\mathcal{M}}\left(\int_{-h}^{z} \mathbf{v}(x, y, \xi, t) d \xi-\frac{1}{h} \int_{-h}^{0} \int_{-h}^{z} \mathbf{v}(x, y, \xi, t) d \xi d z\right) \cdot \nabla_{h}\left(|\widetilde{\mathbf{v}}|^{4} \widetilde{u}\right) d \mathcal{M} \\
& A_{1} \leq C f^{*} \int_{\mathcal{M}}\left(\int_{-h}^{0}|\mathbf{v}(x, y, \xi, t)| d \xi\right)\left|\nabla_{h}\left(|\widetilde{\mathbf{v}}|^{4} \widetilde{u}\right)\right| d \mathcal{M} \\
& A_{1} \leq C \int_{\mathcal{M}}\left(\int_{-h}^{0}|\mathbf{v}(x, y, \xi, t)| d \xi\right)\left|\nabla_{h} \widetilde{\mathbf{v}}\right||\widetilde{\mathbf{v}}|^{4} d \mathcal{M}
\end{aligned}
$$


But, since $\mathcal{M}=\mathcal{M}^{\prime} \times(-h, 0)$, one can write:

$$
\begin{aligned}
& A_{1} \leq C \int_{\mathcal{M}^{\prime}}\left(\int_{-h}^{0}|\mathbf{v}(x, y, \xi, t)| d \xi\right)\left(\int_{-h}^{0}\left|\nabla_{h} \widetilde{\mathbf{v}}\right||\widetilde{\mathbf{v}}|^{2}|\widetilde{\mathbf{v}}|^{2} d z\right) d \mathcal{M}^{\prime}, \\
& A_{1} \leq C\|\overline{|\mathbf{v}|}\|_{L^{4}\left(\mathcal{M}^{\prime}\right)}\left(\int_{\mathcal{M}}\left|\nabla_{h} \widetilde{\mathbf{v}}\right|^{2}|\widetilde{\mathbf{v}}|^{4} d \mathcal{M}\right)^{1 / 2}\left(\int_{\mathcal{M}^{\prime}}\left(\int_{-h}^{0}|\widetilde{\mathbf{v}}|^{4} d z\right)^{2} d \mathcal{M}^{\prime}\right)^{1 / 4} .
\end{aligned}
$$

Using an integral version of Minkowsky inequality for the $L^{2}$ space and a Sobolev and Ladyzhenskaya's inequality in $\mathbb{R}^{2}$, we have a bound on the square of the last term:

$$
\begin{aligned}
\left(\int_{\mathcal{M}^{\prime}}\left(\int_{-h}^{0}|\widetilde{\mathbf{v}}|^{4} d z\right)^{2} d \mathcal{M}^{\prime}\right)^{1 / 2} & \leq C \int_{-h}^{0}\left(\int_{\mathcal{M}^{\prime}}|\widetilde{\mathbf{v}}|^{8} d \mathcal{M}^{\prime}\right)^{1 / 2} d z \\
& \leq C \int_{-h}^{0}\|\widetilde{\mathbf{v}}\|_{L^{6}\left(\mathcal{M}^{\prime}\right)}^{3}\left(\left\|\nabla_{h} \widetilde{\mathbf{v}}\right\|_{L^{2}\left(\mathcal{M}^{\prime}\right)}+\|\widetilde{\mathbf{v}}\|_{L^{2}\left(\mathcal{M}^{\prime}\right)}\right) d z \\
& \leq C\|\widetilde{\mathbf{v}}\|_{6}^{3}\left(\left\|\nabla_{h} \widetilde{\mathbf{v}}\right\|_{2}+\|\widetilde{\mathbf{v}}\|_{2}\right)
\end{aligned}
$$

and

$$
\left(\int_{\mathcal{M}^{\prime}}\left(\int_{-h}^{0}|\widetilde{\mathbf{v}}|^{4} d z\right)^{2} d \mathcal{M}^{\prime}\right)^{1 / 4} \leq C\|\widetilde{\mathbf{v}}\|_{6}^{3 / 2}\left(\left\|\nabla_{h} \widetilde{\mathbf{v}}\right\|_{2}^{1 / 2}+\|\widetilde{\mathbf{v}}\|_{2}^{1 / 2}\right) .
$$

We also study the first term of inequality (3.7) with the same arguments:

$$
\|\overline{|\mathbf{v}|}\|_{L^{4}\left(\mathcal{M}^{\prime}\right)} \leq\|\overline{|\mathbf{v}|}\|_{L^{2}\left(\mathcal{M}^{\prime}\right)}^{1 / 2}\left\|\nabla_{h} \overline{|\mathbf{v}|}\right\|_{L^{2}\left(\mathcal{M}^{\prime}\right)}^{1 / 2} \leq C\|\mathbf{v} \mid\|_{2}^{1 / 2}\left\|\nabla_{h} \mathbf{v}\right\|_{2}^{1 / 2}
$$

We replace inequalities (3.8)-(3.9) in (3.7) and obtain:

$$
\begin{aligned}
& A_{1} \leq C\|\mathbf{v}\|_{2}^{1 / 2}\left\|\nabla_{h} \mathbf{v}\right\|_{2}^{1 / 2}\left(\int_{\mathcal{M}}\left|\nabla_{h} \widetilde{\mathbf{v}}\right|^{2}|\widetilde{\mathbf{v}}|^{4} d \mathcal{M}\right)^{1 / 2}\|\widetilde{\mathbf{v}}\|_{6}^{3 / 2}\left(\left\|\nabla_{h} \widetilde{\mathbf{v}}\right\|_{2}^{1 / 2}+\|\widetilde{\mathbf{v}}\|_{2}^{1 / 2}\right), \\
& A_{1} \leq C\|\mathbf{v}\|_{2}^{2}\left\|\nabla_{h} \mathbf{v}\right\|_{2}^{2}+\frac{\mu_{\mathbf{v}}}{24} \int_{\mathcal{M}}\left|\nabla_{h} \widetilde{\mathbf{v}}\right|^{2}|\widetilde{\mathbf{v}}|^{4} d \mathcal{M}+C\|\widetilde{\mathbf{v}}\|_{6}^{6}\left(\left\|\nabla_{h} \widetilde{\mathbf{v}}\right\|_{2}^{2}+\|\widetilde{\mathbf{v}}\|_{2}^{2}\right) .
\end{aligned}
$$

For the second term $A_{2}$, we start with an integration by parts:

$$
A_{2}=-\int_{\mathcal{M}}\left(f^{*} \int_{z}^{0} u(x, y, \xi, t) d \xi-\frac{f^{*}}{h} \int_{-h}^{0}\left(\int_{z}^{0} u(x, y, \xi, t) d \xi\right) d z\right) \nabla \cdot\left(|\widetilde{\mathbf{v}}|^{4} \widetilde{\mathbf{v}}\right) d \mathcal{M}
$$

This expression is exactly the same as the one of $A_{1}$ if we replace $u$ by $\mathbf{v}$. We directly have:

$$
A_{2} \leq C\|\overline{|u|}\|_{L^{4}\left(\mathcal{M}^{\prime}\right)}\left(\int_{\mathcal{M}}\left|\nabla_{h} \widetilde{\mathbf{v}}\right|^{2}|\widetilde{\mathbf{v}}|^{4} d \mathcal{M}\right)^{1 / 2}\left(\int_{\mathcal{M}^{\prime}}\left(\int_{-h}^{0}|\widetilde{\mathbf{v}}|^{4} d z\right)^{2} d \mathcal{M}^{\prime}\right)^{1 / 4}
$$

and

$$
A_{2} \leq C\|u\|_{2}^{2}\left\|\nabla_{h} u\right\|_{2}^{2}+\frac{\mu_{\mathbf{v}}}{24} \int_{\mathcal{M}}\left|\nabla_{h} \widetilde{\mathbf{v}}\right|^{2}|\widetilde{\mathbf{v}}|^{4} d \mathcal{M}+C\|\widetilde{\mathbf{v}}\|_{6}^{6}\left(\left\|\nabla_{h} \widetilde{\mathbf{v}}\right\|_{2}^{2}+\|\widetilde{\mathbf{v}}\|_{2}^{2}\right) .
$$

Gathering estimates (3.6), (3.10) and (3.11), we get

$$
\begin{array}{r}
\frac{1}{6} \frac{d\|\widetilde{\mathbf{v}}\|_{6}^{6}}{d t}+\frac{\mu_{\mathbf{v}}}{12} \int_{\mathcal{M}}\left|\nabla_{h} \widetilde{\mathbf{v}}\right|^{2}|\widetilde{\mathbf{v}}|^{4} d \mathcal{M}+\frac{\nu_{\mathbf{v}}}{6} \int_{\mathcal{M}}\left|\widetilde{\mathbf{v}}_{z}\right|^{2}|\widetilde{\mathbf{v}}|^{4} d \mathcal{M} \\
\leq C\|\widetilde{\mathbf{v}}\|_{6}^{6}\left(\|\overline{\mathbf{v}}\|_{2}^{2}\left\|\nabla_{h} \overline{\mathbf{v}}\right\|_{2}^{2}+\left\|\nabla_{h} \widetilde{\mathbf{v}}\right\|_{2}^{2}+\|\widetilde{\mathbf{v}}\|_{2}^{2}\right) \\
+C\|\bar{T}\|_{2}^{2}\left\|\nabla_{h} \bar{T}\right\|_{2}^{2}+C\|u\|_{2}^{2}\left\|\nabla_{h} u\right\|_{2}^{2}+C\|\mathbf{v}\|_{2}^{2}\left\|\nabla_{h} \mathbf{v}\right\|_{2}^{2} \\
+
\end{array}
$$


Thanks to Gronwall lemma (as the bounds on $\mathbf{U}$ are uniform, we can apply uniform Gronwall lemma as in [PTZ08]), we obtain:

$$
\|\widetilde{\mathbf{v}}(t)\|_{6} \leq K \text { for all } t \geq t_{0}+r
$$

where $K$ is a constant that does not depend on the initial data.

The equation for $T$ is not modified by the Coriolis terms, it has the same structure as for the classical Primitive Equations. So we will obtain exactly the same estimates as in [PTZ08], namely a time-uniform bound on the $L^{6}$ norm of the temperature.

\subsection{3 $H^{1}$ estimates on the horizontal velocity}

Lemma 3.2 Let $\mathbf{v}$ be a solution of system (3.3). Then, for all $t \geq t_{0}+r$, we have a time-uniform bound on the $H^{1}$ norm of $\mathbf{v}$.

Proof. To have $H^{1}$ estimates, or more precisely $L^{2}$ estimates on the tridimensional gradient of the horizontal velocity, we proceed as follows: first, we get estimates on $\nabla_{h} \overline{\mathbf{v}}$, then we obtain a bound on the vertical derivative of $\mathbf{v}$ and finally we are able to write a result on $\nabla_{h} \mathbf{v}$.

We start multiplying equation (3.4a) by $-\Delta_{h} \overline{\mathbf{v}}$ and obtain:

$$
\begin{aligned}
\frac{1}{2} \frac{d\left\|\nabla_{h} \overline{\mathbf{v}}\right\|_{2}^{2}}{d t}+\frac{\mu_{\mathbf{v}}}{2}\left\|\Delta_{h} \overline{\mathbf{v}}\right\|_{2}^{2} & +A_{3}-A_{4} \\
& \leq C\|\overline{\mathbf{v}}\|_{2}^{2}\left\|\nabla_{h} \overline{\mathbf{v}}\right\|_{2}^{4}+C\left\|\nabla_{h} \widetilde{\mathbf{v}}\right\|_{2}^{2}+C \int_{\mathcal{M}}|\widetilde{\mathbf{v}}|^{4}\left|\nabla_{h} \widetilde{\mathbf{v}}\right|^{2} d \mathcal{M}+C\|\overline{\mathbf{v}}\|_{2}^{2},
\end{aligned}
$$

Here again, $A_{3}$ and $A_{4}$ are the two contributions of the new Coriolis terms:

$$
\begin{aligned}
& A_{3}:=f^{*} \int_{\mathcal{M}^{\prime}} \bar{w}\left(-\Delta_{h} \bar{u}\right) d \mathcal{M}^{\prime} \\
& A_{4}:=\frac{f^{*}}{h} \int_{\mathcal{M}^{\prime}} \nabla_{h}\left(\int_{-h}^{0}\left(\int_{z}^{0} u(x, y, \xi, t) d \xi\right) d z\right)\left(-\Delta_{h} \overline{\mathbf{v}}\right) d \mathcal{M}^{\prime}
\end{aligned}
$$

In order to find bounds on $A_{3}$, we replace $w$ by its expression (3.2):

$$
\begin{aligned}
& A_{3}=-\frac{f^{*}}{h} \int_{\mathcal{M}^{\prime}}\left(\int_{-h}^{0} \int_{-h}^{z} \nabla_{h} \cdot \mathbf{v}(x, y, \xi, t) d \xi d z\right)\left(-\Delta_{h} \bar{u}\right) d \mathcal{M}^{\prime}, \\
& A_{3} \leq C \int_{\mathcal{M}^{\prime}}\left(\int_{-h}^{0}\left|\nabla_{h} \cdot \mathbf{v}\right| d z\right)\left|\Delta_{h} \bar{u}\right| d \mathcal{M}^{\prime}, \\
& A_{3} \leq C\left(\int_{\mathcal{M}^{\prime}}\left(\int_{-h}^{0}\left|\nabla_{h} \cdot \mathbf{v}\right| d z\right)^{2} d \mathcal{M}^{\prime}\right)^{1 / 2}\left(\int_{\mathcal{M}^{\prime}}\left|\Delta_{h} \bar{u}\right|^{2} d \mathcal{M}^{\prime}\right)^{1 / 2} .
\end{aligned}
$$

Applying Cauchy-Schwarz inequality again, one can write:

$$
\int_{\mathcal{M}^{\prime}}\left(\int_{-h}^{0}\left|\nabla_{h} \cdot \mathbf{v}\right| d z\right)^{2} d \mathcal{M}^{\prime} \leq C \int_{\mathcal{M}^{\prime}} \int_{-h}^{0}\left|\nabla_{h} \cdot \mathbf{v}\right|^{2} d z d \mathcal{M}^{\prime}=C\left\|\nabla_{h} \cdot \mathbf{v}\right\|_{2}^{2},
$$


so that

$$
A_{3} \leq C\left\|\nabla_{h} \mathbf{v}\right\|_{2}\left\|\Delta_{h} \bar{u}\right\|_{2} \leq C\left\|\nabla_{h} \mathbf{v}\right\|_{2}^{2}+\frac{\mu_{\mathbf{v}}}{8}\left\|\Delta_{h} \overline{\mathbf{v}}\right\|_{2}^{2} .
$$

For the term $A_{4}$, remark that it is the same as $A_{3}$ replacing $u$ by v. We also obtain:

$$
A_{4} \leq C\left\|\nabla_{h} \mathbf{v}\right\|_{2}^{2}+\frac{\mu_{\mathbf{v}}}{8}\left\|\Delta_{h} \overline{\mathbf{v}}\right\|_{2}^{2}
$$

Combining equations (3.13), (3.14), (3.15) and thanks to the $L^{2}$ estimates, we can apply uniform Gronwall lemma to get:

$$
\left\|\nabla_{h} \overline{\mathbf{v}}(t)\right\|_{2} \leq K \text { for all } t \geq t_{0}+r .
$$

The next step is to deduce estimates for $\mathbf{v}_{z}$, the vertical derivative of $\mathbf{v}$ (from now on we do not need to distinguish between the mean value of $\mathbf{v}$ and its oscillating part anymore). Let us derive equation $(3.3 \mathrm{a})$ with respect to $z$ :

$$
\begin{aligned}
\frac{\partial \mathbf{v}_{z}}{\partial t}+\left(\mathbf{v}_{z} \cdot \nabla_{h}\right) \mathbf{v}+\left(\mathbf{v} \cdot \nabla_{h}\right) \mathbf{v}_{z} & -\left(\int_{-h}^{z} \nabla_{h} \cdot \mathbf{v}\right) \frac{\partial \mathbf{v}_{z}}{\partial z}-\left(\nabla_{h} \cdot \mathbf{v}\right) \mathbf{v}_{z} \\
& +f \mathbf{k} \times \mathbf{v}_{z}+f^{*}\left(\begin{array}{c}
w_{z} \\
0
\end{array}\right)+\nabla_{h}\left(\beta_{T} g T+f^{*} u\right)-\mu_{\mathbf{v}} \Delta_{h} \mathbf{v}_{z}-\nu_{\mathbf{v}} \frac{\partial^{2} \mathbf{v}_{z}}{\partial z^{2}}=0
\end{aligned}
$$

We multiply this equation by $\mathbf{v}_{z}$ and integrate over the domain. Following [CT07] and using (3.3c), we obtain:

$$
\frac{1}{2} \frac{d\left\|\mathbf{v}_{z}\right\|_{2}^{2}}{d t}+\frac{\mu_{\mathbf{v}}}{2}\left\|\nabla_{h} \mathbf{v}_{z}\right\|_{2}^{2}+\frac{\nu_{\mathbf{v}}}{2}\left\|\frac{\partial \mathbf{v}_{z}}{\partial z}\right\|_{2}^{2}+A_{5}+A_{6} \leq C\left(\left\|\nabla_{h} \overline{\mathbf{v}}\right\|_{2}^{4}+\|\widetilde{\mathbf{v}}\|_{6}^{4}\right)+C\|T\|_{2}^{2}
$$

The new Coriolis terms are:

$$
\begin{aligned}
A_{5} & :=f^{*} \int_{\mathcal{M}} w_{z} u_{z} d \mathcal{M}=-f^{*} \int_{\mathcal{M}}\left(\nabla_{h} \cdot \mathbf{v}\right) u_{z} d \mathcal{M}, \\
A_{6} & :=f^{*} \int_{\mathcal{M}} \nabla_{h} u \cdot \mathbf{v}_{z} d \mathcal{M} .
\end{aligned}
$$

These two extra-terms do not bring any technical difficulty as

$$
A_{5,6} \leq C\|\mathbf{v}\|_{2}\left\|\nabla_{h} \mathbf{v}_{z}\right\|_{2} \leq C\|\mathbf{v}\|_{2}^{2}+\frac{\mu_{\mathbf{v}}}{8}\left\|\nabla_{h} \mathbf{v}_{z}\right\|_{2}^{2} .
$$

We replace (3.18) in (3.17) and, using the previous estimates on $\|\widetilde{\mathbf{v}}\|_{6}$ (Lemma 3.1 and equation (3.12)) and $\left\|\nabla_{h} \overline{\mathbf{v}}\right\|_{2}$ (equation (3.16)), we can apply once again uniform Gronwall lemma:

$$
\left\|\mathbf{v}_{z}(t)\right\|_{2} \leq K \text { for all } t \geq t_{0}+r
$$

All these estimates enable us to give a bound on the $H^{1}$ norm of the horizontal velocity $\mathbf{v}$. We multiply equation (3.3a) by the Laplacian of the velocity $-\Delta_{h} \mathbf{v}$ and get:

$$
\begin{aligned}
\frac{1}{2} \frac{d\left\|\nabla_{h} \mathbf{v}\right\|_{2}^{2}}{d t}+\frac{\mu_{\mathbf{v}}}{2}\left\|\Delta_{h} \mathbf{v}\right\|_{2}^{2}+\frac{\nu_{\mathbf{v}}}{2}\left\|\nabla_{h} \mathbf{v}_{z}\right\|_{2}^{2}+A_{7}+A_{8} & \\
& \leq C\left(\|\mathbf{v}\|_{6}^{4}+\left\|\nabla_{h} \mathbf{v}\right\|_{2}^{2}\left\|\mathbf{v}_{z}\right\|_{2}^{2}\right)\left\|\nabla_{h} \mathbf{v}\right\|_{2}^{2}+C\left\|\nabla_{h} T\right\|_{2}^{2},
\end{aligned}
$$


where the new Coriolis terms are defined by:

$$
\begin{aligned}
& A_{7}:=-f^{*} \int_{\mathcal{M}} w \Delta_{h} u d \mathcal{M}=f^{*} \int_{\mathcal{M}}\left(\int_{-h}^{z} \nabla_{h} \cdot \mathbf{v}(x, y, \xi, t) d \xi\right)\left(\Delta_{h} u\right) d \mathcal{M} \\
& A_{8}:=-f^{*} \int_{\mathcal{M}}\left(\nabla_{h} \int_{z}^{0} u(x, y, \xi, t) d \xi\right) \cdot\left(-\Delta_{h} \mathbf{v}\right) d \mathcal{M}
\end{aligned}
$$

In order to replace these terms in equation (3.20), we use the following bounds:

$$
A_{7,8} \leq C\left\|\nabla_{h} \mathbf{v}\right\|_{2}\left\|\Delta_{h} \mathbf{v}\right\|_{2} \leq \frac{\mu_{\mathbf{v}}}{8}\left\|\Delta_{h} \mathbf{v}\right\|_{2}^{2}+C\left\|\nabla_{h} \mathbf{v}\right\|_{2}^{2}
$$

and obtain with Gronwall lemma:

$$
\left\|\nabla_{h} \mathbf{v}(t)\right\|_{2} \leq K \text { for all } t \geq t_{0}+r
$$

which gives us a uniform bound on the $H^{1}$ norm of $\mathbf{v}$.

\subsubsection{Main result}

We also have the classical bound on the $H^{1}$ norm of the temperature, as the Coriolis terms do not appear in equation (3.3b).

These $H^{1}$ bounds on the velocity and the temperature give us the global existence, uniqueness and continuous dependency on initial data of the strong solution to the QHPE (2.7) with the same arguments as [CT07]:

Theorem 3.2 Let $\mathbf{v}_{0} \in V_{1}, T_{0} \in V_{2}$ and $t_{\max }>0$ given. Then there exists a unique strong solution $(\mathbf{v}, T)$ of the system (3.3) on the interval $\left[0, t_{\max }\right]$ which depends continuously on the initial data.

Furthermore, thanks to the uniform bounds, there exist absorbing balls for the solution $(\mathbf{v}, T)$ in $H^{1}(\mathcal{M})$.

Acknowledgement The last two authors are very grateful to Prof. Roger Temam for the fruitful discussions related to this work, together with his help and support over the past years. This work is partially supported by the ANR and NSF under Grants ANR-06-BLAN0306-01 and NSF-DMS-0906440, and by the Research Fund of Indiana University.

\section{References}

[Bur91] Alewyn P. Burger. The potential vorticity equation: from planetary to small scale. Tellus, 43A:191-197, 1991.

[CR94] Benoit Cushman-Roisin. Introduction to Geophysical Fluid Dynamics. Prentice-Hall, 1994.

[CT07] Chongsheng Cao and Edriss S. Titi. Global well-posedness of the three-dimensional viscous primitive equations of large scale ocean and atmosphere dynamics. Ann. of Math., 166(2):245-267, 2007.

[DS05] Paul J. Dellar and Rick Salmon. Shallow water equations with a complete coriolis force and topography. Physics of Fluids, 17(10):106601, 2005.

[Eck60] Carl Eckart. Hydrodynamics of oceans and atmospheres. Pergamon Press, New York, 1960.

[Gil82] Adrian E. Gill. Atmosphere-Ocean Dynamics. New York: Academic Press, 1982.

[Kob07] Georgij M. Kobelkov. Existence of a solution "in the large" for ocean dynamics equations. J. Math. Fluid Mech., 9(4):588-610, 2007.

[KZ07] Igor Kukavica and Mohammed Ziane. On the regularity of the primitive equations of the ocean. Nonlinearity, 20:2739-2753, 2007. 
[LR08] Carine Lucas and Antoine Rousseau. New developments and cosine effect in the viscous shallow water and quasi-geostrophic equations. Multiscale Modeling and Simulations, 7(2):793-813, 2008.

[LR10] Carine Lucas and Antoine Rousseau. Cosine effect in ocean models. Discrete Contin. Dyn. Syst. Series B, 13(4):841-857, 2010.

[LTW92a] Jacques-Louis Lions, Roger Temam, and Shouhong Wang. New formulations of the primitive equations of atmosphere and applications. Nonlinearity, 5(2):237-288, 1992.

[LTW92b] Jacques-Louis Lions, Roger Temam, and Shouhong Wang. On the equations of the large-scale ocean. Nonlinearity, 5(5):1007-1053, 1992.

[Mad08] Gurvan Madec. NEMO ocean engine. Note du Pole de modélisation, Institut Pierre-Simon Laplace (IPSL), France, No 27 ISSN No 1288-1619 edition, 2008.

[MDIL99] Gurvan Madec, Pascale Delecluse, Maurice Imbard, and Claire Lévy. OPA 8.1 Ocean General Circulation Model reference manual. Note du Pole de modélisation, Institut Pierre-Simon Laplace (IPSL), France, 1999.

[Ped87] Joseph Pedlosky. Geophysical fluid dynamics, 2nd edition. Springer, 1987.

[Phi66] Norman A. Phillips. The equations of motion for a shallow rotating atmosphere and the "traditional approximation". Journal of the atmospheric sciences, 23:626-628, 1966.

[Phi68] Norman A. Phillips. Reply (to George Veronis). Journal of the atmospheric sciences, 25:1155-1157, 1968.

[PTZ08] Madalina Petcu, Roger Temam, and Mohammed Ziane. Some mathematical problems in geophysical fluid dynamics. In P. G. Ciarlet, editor, Handbook of Numerical Analysis. Special volume on Computational Methods for the Oceans and the Atmosphere, volume XIV. Elsevier, Amsterdam, 2008.

[SM05] Alexander F. Shchepetkin and James C. McWilliams. The regional oceanic modeling system (ROMS): a split-explicit, free-surface, topography-following-coordinate oceanic model. Ocean Modelling, 9(4):347-404, 2005.

[Ver68] George Veronis. Comments on phillips proposed simplification of the equations of motion for a shallow rotating atmosphere. Journal of the atmospheric sciences, 25:1154-1155, 1968.

[Wan70] Roald K. Wangsness. Comments on "the equations of motion for a shallow rotating atmosphere and the 'traditionnal approximation'". Journal of the atmospheric sciences, 27:504-506, 1970.

[WB95] Andy A. White and R. A. Bromley. Dynamically consistent, quasi-hydrostatic equations for global models with a complete representation of the coriolis force. Q. J. R. Meteorol. Soc., 121:399-418, 1995.

[WHRS05] Andy A. White, Brian J. Hoskins, Ian Roulstone, and Andrew Staniforth. Consistent approximate models of the global atmosphere: shallow, deep, hydrostatic, quasi-hydrostatic and non-hydrostatic. $Q . J$. R. Meteorol. Soc., 131:2081-2107, 2005. 